Cardenete, Manuel Alejandro y Delgado, María del Carmen. Análisis de la estructura de la economía georgiana.

\title{
ANÁLISIS DE LA ESTRUCTURA DE LA ECONOMÍA GEORGIANA
}

\section{ANALYSIS OF THE STRUCTURE OF THE GEORGIAN ECONOMY}

\author{
MANUEL ALEJANDRO CARDENETE FLORES ${ }^{1}$ \\ European Commission \\ Joint Research Centre-Institute for Prospective Technological Studies \\ Manuel-Alejandro.Cardenete@ec.europa.eu \\ Universidad Pablo de Olavide \\ macardenete@upo.es
}

MARÍA DEL CARMEN DELGADO LÓPEZ Universidad Pablo de Olavide mcdellop@upo.es

Fecha recepción: 7 de octubre de 2011 Fecha aceptación: 6 de diciembre de 2011

\section{RESUMEN}

En este trabajo ${ }^{2}$ se pretende analizar la estructura de la economía georgiana para el año 2007. Para ello se utiliza la Matriz de Contabilidad Social de este país para el año objeto de estudio. Se analizarán los sectores clave de la economía, se realizará una descomposición de multiplicadores contables en sus tres efectos (directo, indirecto e inducido), y por último se realizarán dos simulaciones; por un lado se analizará cómo reaccionan los distintos sectores productivos en la región debido a la transferencia de fondos recibidos por parte de las USAID, y por otro lado, se analizará la distribución de los ingresos y los gastos de los consumidores georgianos. Los resultados obtenidos muestran los sectores de actividad más importantes del país, entre los que se encuentran, Agricultura, Manufacturas y Comercio así como los que menos repercusión e importancia tienen como pueden ser Minería, Educación e Instituciones Gubernamentales.

PALABRAS CLAVE: Matrices de Contabilidad Social, Análisis InputOutput, Multiplicadores Lineales.

\footnotetext{
${ }^{1}$ El primer autor agradece la financiación recibida de los proyectos MICINN-ECO2009-11857 y SGR2009-578I y SEJ479, el segundo autor al Proyecto 092-2011 del Centro de Estudios Andaluces. La opinión, análisis y resultados son responsabilidad de los autores y no implican la posición de la Comisión Europea.

${ }^{2}$ Los cálculos de esta trabajo se han realizado con el programa SIMSIPSAM (v.1.1.1, Juan Carlos Parra \& Quentin Wodon, World Bank)
} 
Cardenete, Manuel Alejandro y Delgado, María del Carmen. Análisis de la estructura de la economía georgiana.

JEL: D57, D58, Q20, R13, R15.

\section{ABSTRACT}

This paper analyzes the economic structure of the Georgian economy by 2007. Using the Social Accounting Matrix of this country for that particular year, authors analyze the key sectors of the economy. There can be found an accounting decomposition of multipliers in their three effects (direct, indirect and induced), and finally, the analysis of the reaction of different productive sectors in the region to the transfer of funds received by the USAID and the distribution of income and expenditure of Georgians consumers is run. The results show the most important sectors of the country, among them are to be included: Agriculture, Manufacturing and Trade, as well as the sectors of minor impact and importance which are in the case Mining, Education and Government Institutions.

KEYWORDS: Social Accounting Matrix, Input - Output Analysis, Linear Multipliers.

\section{INTRODUCCIÓN}

Una de las cuestiones más importantes de una economía es conocer su estructura productiva. Para cualquier decisor político es clave la identificación de los sectores productivos capaces de impulsar la economía del país en cuestión. Por lo tanto, su identificación se convierte en un objetivo fundamental, tanto para el crecimiento de las variables macroeconómicas como el PIB, el VAB o como en el aumento del número de empleos. Por ello la evolución que las distintas ramas de actividad puedan ir teniendo a lo largo del tiempo, esto es, sectores productivos que dejan de dinamizar la economía o por el contrario, sectores que se dinamizan e impulsan al resto de la economía, deben ser tenidos en cuenta y perfectamente identificados.

Este trabajo trata de analizar la economía del área geográfica de Georgia para el año 2007. Georgia se sitúa en la costa oriental del Mar Negro. El Cáucaso frontera natural entre Asía y Europa, marca el carácter montañoso del relieve. Es un pequeño país que cuenta con una superficie de $69.700 \mathrm{~km}^{2}$ y una población de 4.370.000 habitantes. Para el año objeto de estudio Georgia cuenta con una población activa de 1.965 .300 , una tasa de paro de $13,3 \%$ y un PIB de 10.117,9 millones \$US.

En el análisis que se llevará a cabo, se estudiaran los sectores económicos clave así como las relaciones más importantes entre estos sectores y la importancia de la región en términos de empleo. Se realizará también una descomposición de los multiplicadores contables que servirá para conocer las relaciones más importantes entre los diferentes sectores productivos de la economía georgiana.

Una vez realizado el análisis cualitativo y cuantitativo de la región se realizarán dos simulaciones para obtener más información adicional: por una parte se simulará cómo reaccionaría la variable macroeconómica PIB ante 
Cardenete, Manuel Alejandro y Delgado, María del Carmen. Análisis de la estructura de la economía georgiana.

transferencias por parte de la Agencia de Estados Unidos para el Desarrollo Internacional (USAID), y por otra parte se analizará el porcentaje de distribución de ingresos y gastos de los distintos grupos de consumidores.

El trabajo se dividirá en una parte teórica donde se explicará la metodología necesaria para el análisis objeto de estudio así como la base de datos utilizada y una segunda parte donde se expondrán e interpretarán los resultados obtenidos presentados con tablas de datos y gráficos.

\section{METODOLOGÍA}

Las tablas input-output (TIO) permiten un análisis estructural de la composición de la economía y el sistema productivo en su conjunto. Este análisis, aunque de una forma estática en cada período, se analizará en distintos momentos sucesivos del tiempo, por lo que podemos considerarlo como estática comparativa evolutiva, muy cercana a la dinámica económica.

Basada en la lógica de la tablas input - output, las matrices de contabilidad social (MCS) o SAM (Social Accounting Matrix) en la terminología anglosajona, intentan corregir y superar algunas de las limitaciones de las bases de datos convencionales usadas para el análisis económico. Por este motivo se puede decir que la MCS es una importante base de datos, organizada en forma de tabla de doble entrada, donde está recogida información económica y social de las transacciones habidas entre todos los agentes económicos.

La diferencia al trabajar con una MCS en lugar de con un TIO radica en las limitaciones de una TIO para crear herramientas de análisis más complejas, como pueden ser entre otros modelos de multiplicadores o modelos computables de equilibrio general (MEGA). Con estas herramientas se pueden realizar distintas simulaciones y estudiar posibles impactos de cambios en las variable exógenas de una determinada economía como por ejemplo cambios en las tarifas o en los precios internacionales.

El uso de las Matrices de Contabilidad Social fue iniciado por Stone (1962) que publicaron una MCS para el Reino Unido. Sin embargo, dada su utilidad para conocer las relaciones intersectoriales de la economía y la distribución de la renta, las primeras MCS fueron elaboradas para países en vías de desarrollo con la finalidad de poner en marcha programas que supusieran una reducción de la pobreza en estos países. Entre otras, hay que destacar la MCS de Sri Lanka elaborada por Pyatt (1977) por el impulso que se dio en este campo y sus aplicaciones, con especial referencia al análisis de multiplicadores Pyatt y Round, (1979).

Una MCS recoge información económica y social relevante de todos los agentes económicos, manifestándose en todas aquellas transacciones realizadas entre los mismos durante un período de tiempo; transacciones que describen operaciones de producción, de distribución y utilización de la renta y de acumulación, tanto dentro de la propia economía como aquellas realizadas con el resto del mundo. 
Cardenete, Manuel Alejandro y Delgado, María del Carmen. Análisis de la estructura de la economía georgiana.

Una MCS amplía la información contenida en una tabla input-output, ya que, además de incluir a ésta, incluyen todos los flujos entre el valor añadido y la demanda final. Por tanto, en una MCS queda reflejado el flujo circular de la renta de una economía.

Si nos fijamos es la figura 1 podemos ver que son las empresas las que producen los bienes y servicios utilizando los factores productivos suministrados por las economías domésticas. Para ello, las empresas pagan por el uso de estos factores, lo que constituyen las rentas de las economías domésticas. Estas rentas son utilizadas para la adquisición de bienes y servicios. Sin embargo, en una economía con sector público, éste detrae de los agentes económicos privados un flujo monetario (impuestos), retornado en forma de bienes públicos, transferencias y subvenciones (gasto público). Las relaciones con el sector exterior hacen que existan unos flujos monetarios de entrada (exportaciones) y salida (importaciones). La utilidad de una MCS se encuentra en que todos estos flujos monetarios quedan incorporados en ella.

FIGURA 1: ESQUEMA SIMPLIFICADO DEL FLUJO CIRCULAR DE LA RENTA.

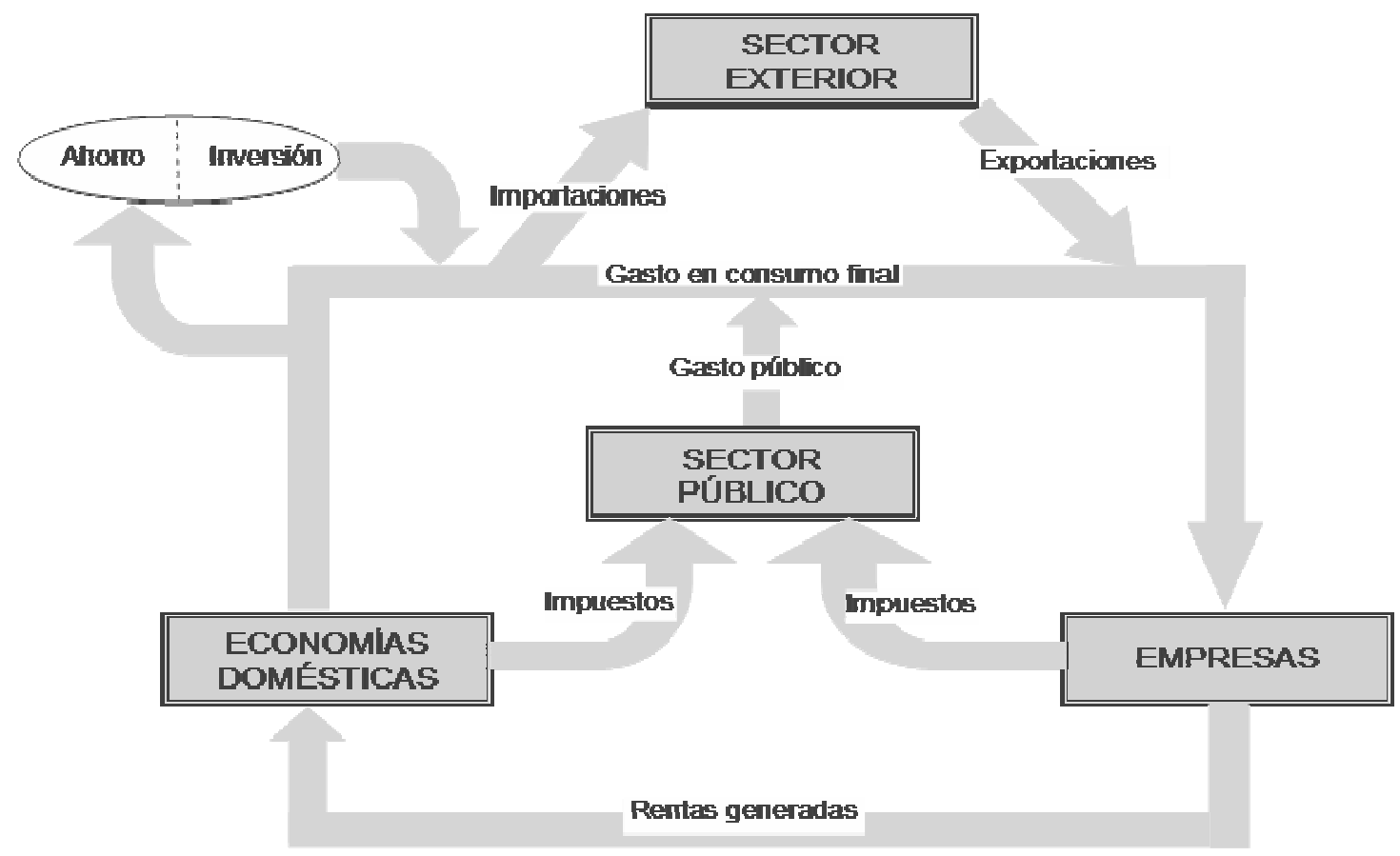

Fuente: Elaboración Propia.

Como en una MCS están representadas todas las transacciones realizadas por los agentes de la economía, se deben cumplir ciertas identidades contables, como que el gasto que realizan los agentes debe ser igual a la renta que han obtenido; esto es, la suma de cada columna debe ser igual a la suma de su correspondiente fila. La estructura de una MCS puede tomar diferentes formas dependiendo de las cuentas que la formen, existiendo, por tanto, una gran flexibilidad. A continuación se expone los bloques o submatrices de una Matriz de Contabilidad Social: 
Cardenete, Manuel Alejandro y Delgado, María del Carmen. Análisis de la estructura de la economía georgiana.

FIGURA 2: ESTRUCTURA ABREVIADA DE UNA MATRIZ DE CONTABILIDAD SOCIAL.

\begin{tabular}{|c|c|c|c|c|c|}
\hline & Producción & $\begin{array}{c}\text { Factores } \\
\text { Productivos }\end{array}$ & $\begin{array}{c}\text { Sectores } \\
\text { Institucionales }\end{array}$ & Inversión & Sector Exterior \\
\hline Producción & $\begin{array}{l}\text { Consumos } \\
\text { Intermedios }\end{array}$ & & $\begin{array}{l}\text { Consumo del } \\
\text { Sector Público } \\
\text { y los Hogares }\end{array}$ & $\begin{array}{c}\text { Formación } \\
\text { Bruta de } \\
\text { Capital }\end{array}$ & Exportaciones \\
\hline $\begin{array}{l}\text { Factores } \\
\text { Productivos }\end{array}$ & $\begin{array}{l}\text { Pagos de VA } \\
\text { a los factores }\end{array}$ & & & & \\
\hline $\begin{array}{l}\text { Sectores } \\
\text { Institucionales }\end{array}$ & $\begin{array}{l}\text { Impuestos s/ } \\
\text { actividades y } \\
\text { bienes y } \\
\text { servicios }\end{array}$ & $\begin{array}{l}\text { Asignación de } \\
\text { ingreso de los } \\
\text { factores a los } \\
\text { Sectores Instit. }\end{array}$ & $\begin{array}{l}\text { Transferencias } \\
\text { corrientes } \\
\text { entre los } \\
\text { Sectores Instit. }\end{array}$ & $\begin{array}{l}\text { Impuestos } \\
\text { s/ bienes } \\
\text { de capital }\end{array}$ & $\begin{array}{c}\text { Transferencias } \\
\text { del Resto del } \\
\text { Mundo }\end{array}$ \\
\hline Inversión & & $\begin{array}{l}\text { Consumo de } \\
\text { capital fijo }\end{array}$ & $\begin{array}{c}\text { Ahorro de los } \\
\text { sectores } \\
\text { institucionales }\end{array}$ & & Ahorro exterior \\
\hline Sector Exterior & Importaciones & & $\begin{array}{c}\text { Transferencias } \\
\text { al Resto del } \\
\text { Mundo }\end{array}$ & & \\
\hline
\end{tabular}

Fuente: Cardenete y Moniche (2001).

- Matriz de consumos intermedios: En esta matriz se recogen las transacciones de bienes y servicios intermedios entre los sectores productivos y entre éstos y las Administraciones Públicas. La suma de las entradas de cada columna indica las compras de bienes intermedios realizadas por cada sector y la suma de la filas representan las ventas efectuadas por cada uno de los sectores. La suma de todas las entradas da como resultado la agregación del consumo intermedio de la economía.

- Matriz de valor añadido: Recoge la descomposición de los recursos utilizados por cada sector productivo. Las partidas incluidas son las siguientes: Trabajo, formada por los Sueldos y Salarios Brutos; Capital, formado por el Consumo de Capital Fijo, el Excedente Neto de Explotación y las Rentas Mixtas; Cotizaciones a la Seguridad Social por parte de los Empleadores.

- Matriz de demanda final: Situada en la parte superior derecha de la Tabla input-output, está formada por las columnas de Consumidores, que representa el gasto total de las economías domésticas, Administración Pública, que recoge el consumo del sector público como agente económico; Ahorro/Inversión, formada por la cuenta Exterior, donde ahora se reflejan las exportaciones a la Unión Europea y al Resto del Mundo.

Estas tres matrices resumen las transacciones entre los agentes de la economía y deben cumplir la identidad contable de que la producción bruta total es igual a la demanda total.

- Matriz de cierre: Esta matriz es la que permite el cierre del flujo circular de la renta de la estructura económica multisectorial que representa una matriz de contabilidad social. Se encuentra en la parte inferior derecha de la MCS y se trata de la parte que realmente completa el marco contable de las TIO. Las tablas de origen y destino describen los procesos de producción y utilización de los bienes y servicios, pero no incorporan las interrelaciones entre el valor añadido y el gasto final. Estas interrelaciones sí aparecen reflejadas en la MCS, lo que permite mostrar en su totalidad el flujo circular de la renta. En las filas y columnas correspondientes a Hogares y Sector Público aparecen por filas los recursos totales para hacer frente a sus gastos en consumo e 
Cardenete, Manuel Alejandro y Delgado, María del Carmen. Análisis de la estructura de la economía georgiana.

inversión. Por columnas se muestran los empleos en gastos finales, pago de impuestos o ahorro.

\section{INFORMACIÓN ESTADÍSTICA}

Los datos necesarios para llevar a cabo el análisis han sido obtenidos de la oficina nacional de estadística de Georgia, a partir de un proyecto de investigación financiado por Millenium Challenge Corporation y TSBC llevado a cabo por Cardenete (2009).

Los datos se encuentran en millones de Lari, moneda georgiana, por lo que se ha realizado una conversión a millones de dólares para poder trabajar con una moneda de ámbito internacional.

A continuación se puede observar la estructura de cuentas de la MCS, dividida en 15 ramas productivas y 19 cuentas más para los sectores institucionales. Se consideraran como endógenas las cuentas de los 15 sectores y además se endogeneizarán, las cuentas de trabajo, capital (16 y 17), quedando el resto como cuentas exógenas.

Resaltar la desagregación de los consumidores en grupos de gasto distintos así como grupos de residencia diferenciados, dependiendo de que su vivienda se encuentre en el campo o en la ciudad.

TABLA 1: ESTRUCTURA DE LA MATRIZ DE CONTABILIDAD SOCIAL DE GEORGIA. AÑo 2007.

\begin{tabular}{|c|l|r|l|}
\hline 1 & Agricultura & 18 & Inversión \\
\hline 2 & Minería y Canteras & 19 & Consumidores Urbanos nivel de gasto bajo \\
\hline 3 & Manufacturas & 20 & Consumidores Rurales nivel de gasto bajo \\
\hline 4 & $\begin{array}{l}\text { Electricidad, Gas, Vapor y Agua } \\
\text { caliente }\end{array}$ & 21 & $\begin{array}{l}\text { Consumidores Urbanos nivel de gasto } \\
\text { medio }\end{array}$ \\
\hline 5 & Turismo & 22 & $\begin{array}{l}\text { Consumidores Rurales nivel de gasto } \\
\text { medio }\end{array}$ \\
\hline 6 & Trasportes y Logística & 23 & Consumidores Urbanos nivel de gasto alto \\
\hline 7 & Correos y Telecomunicaciones & 24 & Consumidores Rurales nivel de gasto alto \\
\hline 8 & Comercio & 25 & Impuesto sobre la renta \\
\hline 9 & Construcción & 26 & Impuesto sobre las ganancias corporativas \\
\hline 10 & Intermediación Financiera & 27 & Impuesto a la propiedad \\
\hline 11 & Servicios comerciales & 28 & IVA \\
\hline 12 & Educación & 29 & Impuesto Selectivo al consumo \\
\hline 13 & Sanidad y Asistencia Social & 30 & Otros Impuestos \\
\hline 14 & $\begin{array}{l}\text { Servicios Comunales, Sociales y } \\
\text { Personales }\end{array}$ & 31 & Aranceles \\
\hline 15 & Instituciones Gubernamentales & 32 & Subvenciones a los productos \\
\hline 16 & Trabajo & 33 & Sector Público \\
\hline 17 & Capital & 34 & Sector Exterior \\
\hline
\end{tabular}

Fuente: Elaboración Propia a partir del trabajo de La Matriz de Contabilidad Social de Georgia 2007 (mineo) 
Cardenete, Manuel Alejandro y Delgado, María del Carmen. Análisis de la estructura de la economía georgiana.

\section{RESULTADOS}

\subsection{Sectores clave: indicadores de análisis estructural}

En la metodología que se aplica en este apartado, se extiende el Modelo de Leontief a Modelos MCS lineales, para evaluar, mediante el cálculo de los multiplicadores, como son los cambios en producción, renta o empleo en las cuentas endógenas a causa de políticas que modifiquen las cuentas exógenas para las ramas productivas de la economía georgiana. La Teoría de multiplicadores fue iniciada por Stone (1978), y Pyatt y Round (1979), desarrollándose posteriormente con trabajos como los de Defourney y Thorbecke (1984). Estos métodos se basan en obtener información, a partir de las matrices inversas derivadas de los modelos de Leontief (1941) y de Ghosh (1958) aplicados a la MCS, sobre la capacidad que tiene un sector de expandir aumentos de demanda o de costes, respectivamente. La metodología se apoya en la combinación de dos tipos de enlaces intersectoriales, los Backward linkages (BL), o vínculos hacia atrás, y los Forward linkages (FL), o vínculos hacia delante. El BL aporta información sobre el efecto que tiene el aumento de la demanda de un sector en la economía, es decir, de donde proceden los inputs que un sector requiere para incrementar su producción. Los FL ofrecen información sobre el efecto en el resto de sectores de cambios en el valor de los inputs primarios, y, por tanto, en la producción, de un sector concreto, es decir, cual es el destino de la producción de un sector y en qué medida afecta al resto la variación en su valoración. En este apartado se desarrolla esta metodología, que se aplicará a la MCS de la economía georgiana para el año 2007, con el objetivo de identificar los sectores clave y las principales interrelaciones de la provincia. Comenzamos con una breve explicación de los Modelos MCS lineales, como extensión del Modelo Leontief, de la siguiente forma: se considera una matriz cuadrada $n \times n$ donde cada fila y cada columna representan una cuenta económica (sectores productivos, consumidores, gobierno, cuenta de capital, etc.) que satisface las igualdades contables de la economía (total renta igual a total gasto). Cada componente $Y_{i j}$ de la matriz representa el flujo bilateral entre la cuenta $i$ y la cuenta $j$. Cada fila de la MCS recoge el total de ingresos que la fila $i$ recibe de las columnas $j$; las columnas muestran el total de renta de la columna $j$ y como se distribuye entre las distintas cuentas $i$. Los coeficientes medios de gasto: $a_{i j}=Y_{i j} / Y_{j}, i, j=1, \ldots, n$., muestran los pagos a la cuenta $i$ por unidad de renta de $j$. De esta definición se puede obtener:

$$
Y_{i}=\sum_{j=1}^{n}\left(Y_{i j} / Y_{j}\right) Y_{j}=\sum_{j=1}^{m} a_{i j} Y_{j}+\sum_{j=m+1}^{m+k} a_{i j} Y_{j} ; \text { donde } n=m+k
$$

Los índices $m$ y $k$ representan la división de las cuentas de la MCS entre endógenas y exógenas, lo que divide la matriz $n x n$ en 4 submatrices: $A_{m m}, A_{m k}$, $A_{k m}$, y $A_{k k} . Y_{m}$ e $Y_{k}$ denotan la renta total de las cuentas endógenas y exógenas respectivamente, por lo que se puede despejar $Y_{m}$ y obtener $Y_{m}=A_{m m} Y_{m}+A_{m k}$ $Y_{k}$, y desde ahí, siguiendo el mismo procedimiento que con la ecuación de Leontief, obtener la matriz de multiplicadores extendidos a partir de $Y_{m}=(I-$ 
Cardenete, Manuel Alejandro y Delgado, María del Carmen. Análisis de la estructura de la economía georgiana.

$\left.A_{m m}\right)^{-1} Z$, siendo $Z$ el vector de las columnas exógenas ${ }^{3}\left(A_{m k} Y_{k}\right)$, y $M=\left(I-A_{m m}\right)^{-}$ 1 la matriz de los multiplicadores extendidos de la MCS, que pueden interpretarse como las necesidades de inputs por incrementos unitarios de gasto o renta (según hablemos de columnas o filas) en una cuenta, de manera similar a la que tiene la conocida como inversa de Leontief, con la diferencia de que sí que capta las relaciones entre la producción, la renta de los factores, la distribución de la renta y la demanda final. Hay que señalar que la selección de $m$ (es decir, la decisión de qué cuentas son endógenas) suele depender del análisis que se vaya a realizar, y en función de eso se decide qué cuentas son las que explican (exógenas) cambios en la renta de otras cuentas (endógenas). Si llamamos $d Z$ a los cambios en el vector de cuentas exógenas, los cambios en la renta de las cuentas endógenas serían:

$$
d Y_{m}=M d Z=M d\left(A_{m k} Y_{k}\right)=M A_{m k} d Y_{k}
$$

La columna i-ésima de $M$ indica las rentas totales generadas en cada una de las cuentas endógenas cuando se produce un flujo unitario de renta desde las instituciones exógenas hacia la cuenta endógena i. A partir de esta interpretación de las columnas de $M$ y normalizando ${ }^{4}$ obtenemos $B L . j$, que analiza los efectos difusión, vínculos hacia atrás o backward linkages.

$$
B L_{j}=\frac{M_{j}}{\frac{1}{n} \sum_{j=1}^{n} M_{j}}
$$

Para determinar los sectores clave necesitamos el otro tipo de enlace, $F L_{i}$., que analiza los vínculos hacia adelante. Para su cálculo, partimos del modelo de precios de Ghosh, que cuantifica el cambio en el output del sector $i$ que ocurriría como consecuencia de un incremento unitario exógeno en los inputs primarios del sector $j$ (o en su precio). A partir de aquí obtenemos este enlace, como una valoración del efecto conjunto sobre todos los sectores, de modificar el valor de los inputs primarios de un sector en particular. Siendo $\delta_{i j}$ los coeficientes técnicos de la inversa ghoshiana:

$$
F L_{i}=\frac{\sum_{j=1}^{n} \delta_{i j}}{\frac{1}{n} \sum_{i=1}^{n} \sum_{j=1}^{n} \delta_{i j}}
$$

\footnotetext{
${ }^{3}$ La submatriz $A_{m k}$ representa cómo se reparten los flujos de renta de las cuentas exógenas entre las cuentas endógenas.

${ }^{4}$ Polo, Roland-Host, y Sancho (1991).

${ }^{5}$ Para normalizar calculamos primero el efecto medio conjunto como suma de los efectos de todos los sectores dividiendo por el número de sectores. A continuación, normalizamos el indicador dividiendo el efecto de cada sector por la media obtenida.
} 
Cardenete, Manuel Alejandro y Delgado, María del Carmen. Análisis de la estructura de la economía georgiana.

Siguiendo a Dietzenbacher (1997), entendemos que cada elemento de la inversa de Ghosh, $\delta_{i j}$, nos dice cuánto tiene que aumentar el valor de la producción del sector $j$, para que sea posible un incremento de una unidad monetaria en el valor añadido en el sector $i$. Por tanto, la suma de la fila i-ésima de la inversa de Ghosh, muestra en cuantos euros tiene que ser incrementado el valor del output de todos los sectores, debido a un incremento de un euro en el valor añadido del sector $i$. Una vez que obtenemos ambos enlaces, su interpretación es como sigue, aquellos sectores con FL superior a uno son sectores con capacidad de dispersión de costes, o lo que es lo mismo, los cambios en la cuantía de su valor añadido afectan más al sistema que la media. Los sectores con $B L$ superior a uno tienen poder de dispersión, es decir, una variación en su producción influye más en el sistema que la media. Ambos índices nos permiten, además, identificar cuáles son los sectores clave de una economía, que se definen como aquellos sectores que tienen tanto poder de dispersión de variaciones en la demanda $(B L,>1)$, cómo capacidad de dispersión de variaciones en los costes $(F L .>1)$. Se les identifica como clave porque estos sectores tienen una alta influencia en la evolución de la actividad económica, debido a que sus ofertas y demandas están en la mayoría de los casos muy repartidas entre las distintas ramas, y además a que suelen ser fuertes demandantes de inputs intermedios. A continuación aplicamos esta metodología a la economía georgiana, partiendo de la MCS de Georgia para el año 2007.

TABLA 2: CLASIFICACIÓN SECTORIAL A PARTIR DE LOS BL Y FL.

\begin{tabular}{|l|l|l|}
\cline { 2 - 3 } \multicolumn{1}{c|}{} & \multicolumn{1}{|c|}{ BL<Promedio $(\mathrm{BL})$} & \multicolumn{1}{c|}{ BL>Promedio $(\mathrm{BL})$} \\
\hline FL<Promedio $(\mathrm{FL})$ & Sectores independientes & Sectores impulsores \\
\hline FL $>$ Promedio $(\mathrm{FL})$ & Sectores base & Sectores clave \\
\hline
\end{tabular}

Fuente: Elaboración Propia.

- Sectores clave: demanda y ofrecen grandes cantidades de inputs intermedios que suelen estar muy repartidas entre las distintas ramas, son una parte muy importante del flujo de toda la economía, al ser fuertemente impulsados por variaciones en cualquier sector, y este impulso afecta a su vez de forma importante al resto del sistema con lo que pueden provocar a un aumento generalizado de la actividad económica.

- Sectores base o estratégicos: son aquellos que presentan una baja demanda de inputs, siendo el destino de su producción preferentemente el uso intermedio, es decir, sirve de input a otros sectores, por lo que sus variaciones, en precios o cantidades, afectan de forma importante al resto.

- Sectores impulsores: son grandes demandantes de inputs intermedios y dada la capacidad que tienen de inducir otras actividades pueden afectar en mayor cuantía al crecimiento global de la economía.

- Sectores independientes: son aquellos que presentan unos encadenamientos hacia delante y hacia atrás por debajo de la media, por lo que afectan y son afectados por el sistema de forma inferior a la media. 
Cardenete, Manuel Alejandro y Delgado, María del Carmen. Análisis de la estructura de la economía georgiana.

En las siguientes tablas podemos ver la clasificación de sectores en los grupos arriba señalados para el año en cuestión:

TABLA 3: CLASIFICACIÓN SECTORIAL.

\begin{tabular}{|c|l|l|}
\hline № Cuenta & \multicolumn{1}{|c|}{ Sectores Productivos } & Clasificación \\
\hline 1 & Agricultura & Clave \\
\hline 2 & Minería y Canteras & Independiente \\
\hline 3 & Manufacturas & Clave \\
\hline 4 & Electricidad, Gas, Vapor y Agua caliente & Clave \\
\hline 5 & Turismo & Impulsor \\
\hline 6 & Transportes y Logística & Clave \\
\hline 7 & Correos y Telecomunicaciones & Clave \\
\hline 8 & Comercio & Clave \\
\hline 9 & Construcción & Clave \\
\hline 10 & Intermediación Financiera & Impulsor \\
\hline 11 & Servicios comerciales & Clave \\
\hline 12 & Educación & Independiente \\
\hline 13 & Sanidad y Asistencia Social & Impulsor \\
\hline 14 & Servicios Comunales, Sociales y Personales & Impulsor \\
\hline 15 & Instituciones Gubernamentales & Independiente \\
\hline
\end{tabular}

Fuente: Elaboración Propia.

Se puede observar que para Georgia en el año 2007 son sectores clave las ramas de Agricultura (1), Manufacturas (3), Producción de Electricidad, gas y vapor de agua caliente (4), Transporte y Logística (6), Correos y Telecomunicaciones (7), Comercio (8), Construcción (9) y Servicios comerciales (11). Los sectores impulsores de la economía georgiana son las ramas de los sectores Turismo (5), Sanidad y Servicios Sociales (13), y la rama de Servicios Comunales, Sociales y Personales (14). Se han identificado como sectores independientes el resto de sectores. Los resultados obtenidos confirman el fuerte peso de la agricultura en la economía georgiana. La agricultura es un rasgo importante de la economía y el país tiene uno de los sectores de agricultura más variados de cualquiera de las antiguas repúblicas soviéticas. Las ramas producción de electricidad, gas y vapor de agua caliente, así como construcción, comercio, servicios comerciales, telecomunicaciones e intermediación financiera, han sufrido un impulso considerable debido a su mejor inclusión en la economía nacional a causa de grandes proyectos de infraestructura, relacionados con el sector hidrocarburos. Por otra parte se observa en los resultados la debilidad de la industria del país, ya que se encuentra entre los sectores independientes, a excepción del sector manufacturas. La poca y muy concentrada producción industrial se debe principalmente al escaso número de empresas. 
Cardenete, Manuel Alejandro y Delgado, María del Carmen. Análisis de la estructura de la economía georgiana.

\subsection{Descomposición de multiplicadores}

En este apartado se completa el análisis sectorial mediante el desarrollo de la metodología de descomposición de multiplicadores a partir de Defourny y Thorbecke (1984) y Pyatt y Round (1985), y a partir de trabajos como los de Polo, Roland - Host y Sancho (1991) para la economía española. Esta metodología nos permite incorporar otros vínculos, además de los ya comentados arriba (interindustriales), que se producen entre las rentas de los factores primarios y las diversas instituciones que forman la demanda final. De donde se puede separar los siguientes efectos:

- Efecto Directo $=(I+A)$, mide el efecto sobre la actividad de un sector de tener que ajustar su producción para satisfacer los nuevos niveles de demanda final.

- Efecto Indirecto = $(M I-I-A)$, mide los ajustes en los niveles de producción de los sectores, en respuesta a las nuevas demandas de inputs, que son necesarias para acomodar el nivel de producción del sector en el que originalmente recae la nueva demanda final.

- Efecto Inducido = $(\mathrm{Ma}-\mathrm{MI})$, mide el impacto que el crecimiento de las rentas ejerce, vía demanda sobre los niveles de actividad.

- Efecto total= Efecto directo + Efecto Indirecto + Efecto inducido.

Con este procedimiento se ha logrado descomponer la matriz de multiplicadores contables en otras tres matrices mediante una expresión aditiva siguiendo a Pyatt y Round (1979). El modelo que se desarrollará en este apartado es un claro ejemplo de las ventajas que tiene el análisis MCS frente al tradicional enfoque Input-Output, dado que éste no considera las interdependencias presentes en el flujo circular de la renta, mientras que las MCS si que incorporan estos flujos, por lo que se captan los efectos de retroalimentación que se producen desde los agentes receptores de rentas hacia todos los sectores. De esta manera será posible valorar también estos efectos derivados del flujo circular de la renta, como el efecto de una variación de rentas sobre los niveles de actividad, denominados efectos inducidos. El análisis de estos efectos, permite superar algunas de las carencias del análisis input-output tradicional. Los multiplicadores lineales de una MCS, al igual que los multiplicadores input-output, estiman los efectos que producen cambios en las variables exógenas sobre las endógenas. Por tanto, realizar un análisis que incluya los efectos inducidos e indirectos, además de los efectos directos, supone un avance a la hora de obtener información más detallada de los mecanismos de interdependencia existentes en una economía. A continuación presentamos los resultados obtenidos para la economía georgiana en el año 2007.

En la tabla 4 se observa como el sector Construcción es el que tiene un efecto directo e indirecto mayor, 1.4987 y 0.3162 respectivamente, significando esto que cada tirón de demanda de la economía este sector genera 1.4987 y en el caso del efecto indirecto la intuición es la misma que la del efecto directo pero en una segunda vuelta porque puede seguir necesitando de ese sector. En el caso del efecto inducido, debido a la endogeneización de las cuentas Trabajo, Capital y Consumo, aumenta la demanda de estas tres cuentas y se traduce en 
Cardenete, Manuel Alejandro y Delgado, María del Carmen. Análisis de la estructura de la economía georgiana.

un aumento de la demanda de todos los sectores, en el caso de Georgia para el año 2007 el sector que más aumenta es Servicios Comerciales.

TABLA 4: Descomposición de LoS EFECTOS totALES EN EFECTOS DIRECTOS, INDIRECTOS E INDUCIDOS (EN PORCENTAJES).

\begin{tabular}{|c|l|c|c|c|c|}
\hline № Cuenta & \multicolumn{1}{|c|}{ Sectores Productivos } & Efecto Total & $\begin{array}{c}\text { Efecto } \\
\text { Directo }\end{array}$ & $\begin{array}{c}\text { Efecto } \\
\text { Indirecto }\end{array}$ & $\begin{array}{c}\text { Efecto } \\
\text { Inducido }\end{array}$ \\
\hline 1 & Agricultura & 2.9858 & 1.2580 & 0.0958 & 1.6320 \\
\hline 2 & Minería y Canteras & 1.8091 & 1.1619 & 0.0813 & 0.5659 \\
\hline 3 & Manufacturas & 1.8864 & 1.2466 & 0.0918 & 0.5481 \\
\hline 4 & Electricidad, Gas, Vapor y Agua caliente & 3.1129 & 1.3837 & 0.1700 & 1.5592 \\
\hline 5 & Turismo & 2.7147 & 1.3025 & 0.1036 & 1.3086 \\
\hline 6 & Transportes y Logística & 2.4448 & 1.2018 & 0.0767 & 1.1664 \\
\hline 7 & Correos y Telecomunicaciones & 3.2169 & 1.2993 & 0.1122 & 1.8054 \\
\hline 8 & Comercio & 3.1681 & 1.3422 & 0.1253 & 1.7006 \\
\hline 9 & Construcción & 3.2474 & 1.4987 & 0.3162 & 1.4325 \\
\hline 10 & Intermediación Financiera & 2.7051 & 1.2625 & 0.0937 & 1.3489 \\
\hline 11 & Servicios comerciales & 3.1522 & 1.2125 & 0.0874 & 1.8522 \\
\hline 12 & Educación & 3.0057 & 1.1746 & 0.0816 & 1.7495 \\
\hline 13 & Sanidad y Asistencia Social & 3.2094 & 1.3469 & 0.1531 & 1.7094 \\
\hline 14 & Servicios Comunales, Sociales y Personales & 3.2981 & 1.3614 & 0.1413 & 1.7955 \\
\hline 15 & Instituciones Gubernamentales & 3.1322 & 1.2521 & 0.1029 & 1.7772 \\
\hline
\end{tabular}

Fuente: Elaboración Propia.

\subsection{Paisaje tridimensional de la economía georgiana}

Para completar este análisis sectorial de la economía georgiana se calculará la Matriz del Producto Multiplicador (MPM), derivada de la MCS, que nos permite analizar las interdependencias sectoriales de esta economía. A partir de M, se definen los elementos de esta matriz como el producto de los multiplicadores $M$ fila (Mi.) y columna (M.j), dividido por un factor de intensidad total, este factor es calculado como la suma de todos los elementos de la matriz M:

$$
\operatorname{MPM}_{i j}=\frac{M_{i} M_{j}}{\sum_{i=1}^{n} \sum_{j=1}^{n} m_{i j}}
$$

A partir de esta matriz MPM elaboramos un paisaje tridimensional (o landscape) de la economía georgiana que permite identificar visualmente cuáles son los sectores que generan un impacto superior a la media en la economía (sectores con poder de dispersión), por cambios en ellos mismos, y cuáles son los sectores que se ven más influidos por cambios en el resto del sistema (sectores con sensibilidad de dispersión), así como la forma en la que interactúan con el resto de ramas del sistema. A continuación se presenta el 
Cardenete, Manuel Alejandro y Delgado, María del Carmen. Análisis de la estructura de la economía georgiana.

gráfico donde se puede ver el paisaje tridimensional de la economía georgiana para el año 2007. Para una mejor visualización de los resultados presentamos las 15 cuentas en orden de importancia en los enlaces intersectoriales.

FIGURA 3: PAISAJE TRIDIMENSIONAL. GEORGIA 2007.

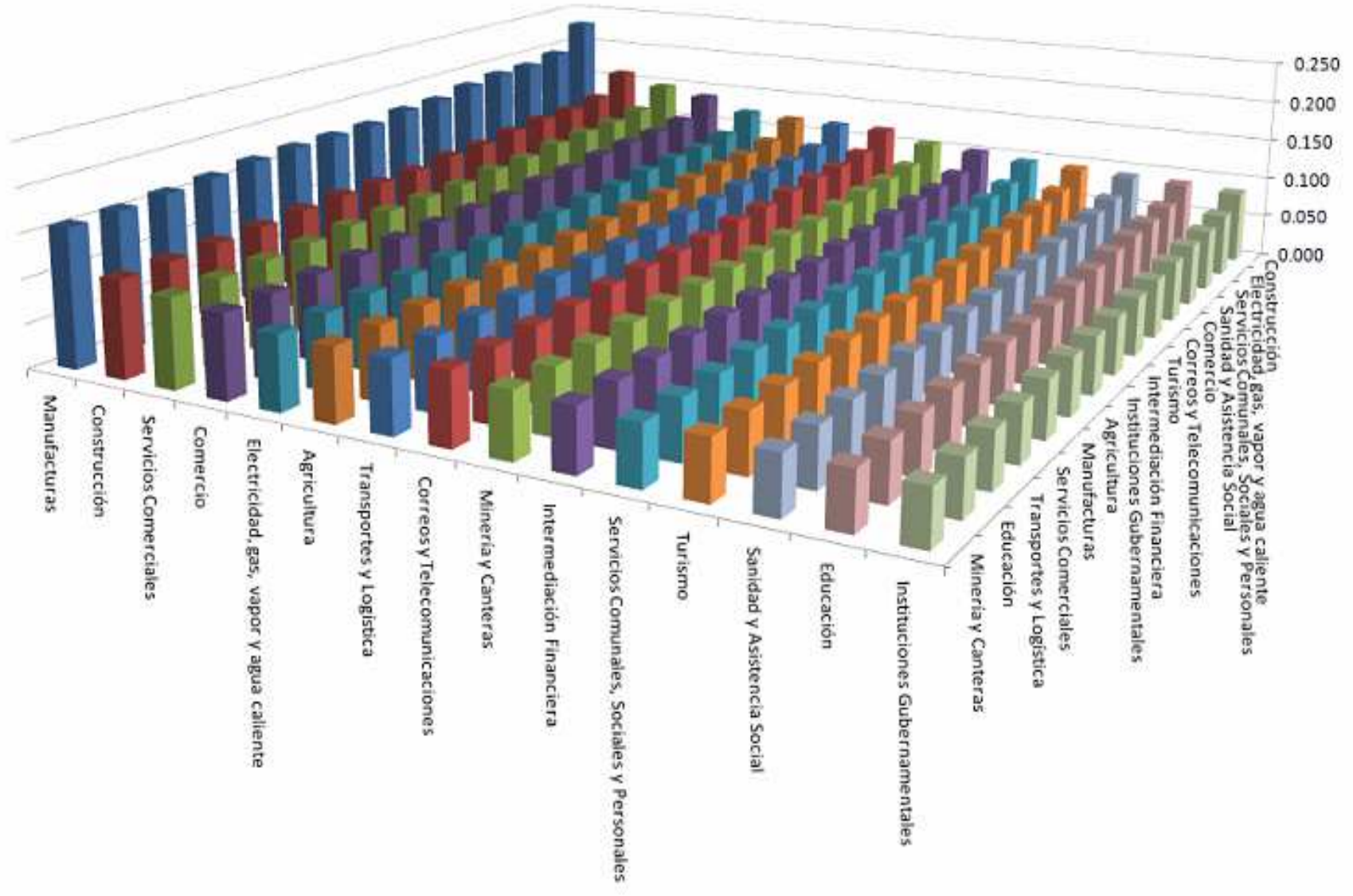

Fuente: Elaboración Propia.

En la Figura 3 se puede comprobar visualmente los resultados obtenidos en el apartado anterior. Se observa como el sector con mayor importancia son Manufacturas (3), seguido de Construcción (9), Servicios Comerciales (11) y Comercio (8). Se ve claramente la poca importancia de la rama de Instituciones Gubernamentales (15) y Educación (12). También se pueden observar los enlaces que adquieren mayor importancia, destacando los vínculos entre las ramas manufacturas (3) con Construcción (9).

\subsection{Multiplicadores de empleo}

A partir de la información obtenida para el país para el año objeto de estudio a continuación se presentan los multiplicadores de empleo obtenidos. Estos multiplicadores nos indican el efecto expansivo de shocks de demanda final, es decir, el grado de sensibilidad de cada sector en términos de empleo a la demanda. El multiplicador de empleo de cada sector vendrá determinado por: 
Cardenete, Manuel Alejandro y Delgado, María del Carmen. Análisis de la estructura de la economía georgiana.

$$
E_{j}=\sum_{i=1}^{n} w_{n+1, i} b_{i j}
$$

Siendo $w_{n+1, i}=Y^{e_{i}} / X_{i}$. $Y^{e_{i}}$ el empleo de cada sector, $\mathrm{X}_{\mathrm{i}}$ el output total del sector i. $b_{i j}$ por su parte, es el elemento ij de la matriz $\mathrm{M}$ de multiplicadores obtenida a partir de la MCS.

TABLA 5: MULTIPLICADOR DE EMPLEO DE LA ECONOMÍA GEORGIANA. AÑo 2007.

\begin{tabular}{|c|l|c|}
\hline № Cuenta & \multicolumn{1}{|c|}{ Sectores Productivos } & Multiplicador \\
\hline 1 & Agricultura & 0.135 \\
\hline 2 & Minería y Canteras & 0.133 \\
\hline 3 & Manufacturas & 0.097 \\
\hline 4 & Electricidad, Gas, Vapor y Agua caliente & 0.250 \\
\hline 5 & Turismo & 0.063 \\
\hline 6 & Transportes y Logística & 0.035 \\
\hline 7 & Correos y Telecomunicaciones & 0.160 \\
\hline 8 & Comercio & 0.150 \\
\hline 9 & Construcción & 0.185 \\
\hline 10 & Intermediación Financiera & 0.152 \\
\hline 11 & Servicios comerciales & 0.214 \\
\hline 12 & Educación & 0.041 \\
\hline 13 & Sanidad y Asistencia Social & 0.085 \\
\hline 14 & Servicios Comunales, Sociales y Personales & 0.090 \\
\hline 15 & Instituciones Gubernamentales & 0.030 \\
\hline Fuente: Elaboración Propia. & \\
\hline
\end{tabular}

En la Tabla 5 se detallan los resultados obtenidos para los multiplicadores de empleo para el año 2007. Estos resultados muestran como los sectores que mejor responden a inyecciones de renta exógena son las ramas de electricidad, gas, vapor y agua caliente (4), que, por cada aumento de una unidad en la demanda final, este sector genera 0,250 y la rama de servicios comerciales (11), que, por cada aumento de una unidad en la demanda final, este sector genera 0,214. Destacar, que las ramas con mayor multiplicador de empleo, coinciden en su mayoría con los detectados sectores clave. Estos resultados pueden explicar en parte que la tasa de desempleo del país se mantenga en niveles no muy elevados.

\subsection{Simulaciones}

En este apartado se llevarán a cabo dos simulaciones distintas. Una primera simulación consistirá en introducir un shock en la economía para comprobar cómo reaccionaría la variable PIB y cuál sería el impacto que tendría en los Papeles de Europa 
Cardenete, Manuel Alejandro y Delgado, María del Carmen. Análisis de la estructura de la economía georgiana.

precios de la economía ante transferencias de la Agencia de Estados Unidos para el Desarrollo Internacional (USAID).

Tomando en cuenta la definición dada por la Agencia en su propia página web "La Agencia de Estados Unidos para el Desarrollo Internacional (USAID) es la agencia federal independiente responsable de planificar y administrar la asistencia económica y humanitaria exterior de los Estados Unidos en todo el mundo, además es la encargada de "administrar" programas de asistencia en 80 países. USAID apoya el crecimiento económico, la salud, la educación, la democracia, la agricultura, la prevención de conflictos y provee asistencia humanitaria en caso de desastres".

Una segunda simulación consistirá en conocer la distribución porcentual de los ingresos y gastos para los seis tipos de consumidores en la MCS. Como se explicaba en el apartado tres, la cuenta consumidores de la MCS objeto de estudio se encuentra desagregada en grupos de gasto distintos así como grupos de residencia diferenciados, dependiendo de que su vivienda se encuentre ubicada en el campo o en la ciudad. Así la cuenta consumidores se encuentra desagregada en seis subcuentas:

- Consumidores Urbanos con nivel de gasto bajo (URBLOWEXP)

- Consumidores Rurales con nivel de gasto bajo (RURALOWEXP)

- Consumidores Urbanos con nivel de gasto medio (URBMEDEXP)

- Consumidores Rurales con nivel de gasto medio (RURAMEDEXP)

- Consumidores Urbanos con nivel de gasto alto (URBHIGHEXP)

- Consumidores Rurales con nivel de gasto alto (RURAHIGHEXP)

A continuación se desarrollarán ambas simulaciones y los resultados obtenidos para las mismas.

\subsubsection{Simulación 1: impacto sectorial en el crecimiento y el precio}

Los sectores productivos pueden ser comparados en términos de su impacto en el crecimiento de al menos dos formas distintas:

Se pueden realizar shocks a todos los sectores, por exactamente la misma cantidad y comparar el impacto en el PIB total, o podemos calcular la elasticidad del PIB de cada sector. Sea $P I B_{i}$ e $Y_{i}$ denotados como el PIB del sector y la oferta total respectivamente, mientras que las variables sin subíndice denotan cifras agregadas. Luego un impacto en el sector j en el agregado del PIB (en porcentaje) se calcula:

$$
\Delta P I B_{j}=\sum_{i} \frac{P I B_{i}}{Y_{j}} M_{i j} \text { Shock/PIB }
$$

de donde, $M_{i j}$ denota la celda $(i, j)$ de la matriz inversa, y en el shock se expresa como un porcentaje del PIB total y se mantiene constante en todos los sectores $j$. El porcentaje de cambio en la oferta de productos básicos $j$ está dado por: 
Cardenete, Manuel Alejandro y Delgado, María del Carmen. Análisis de la estructura de la economía georgiana.

$$
\Delta Y_{j}=M_{i j} \text { Shock } / Y_{j}
$$

La oferta total de la elasticidad del PIB de productos básicos j se puede ahora calcular como:

$$
\varepsilon_{P I B, j}=\Delta P I B_{j} / \Delta Y_{j}
$$

Se puede realizar un ejercicio similar con los precios, en el cuál se puede comparar el impacto de un incremento en el precio de cada sector así como sobre un índice agregado como puede ser el Índice de Precios a la Producción (IPP). Se considera la siguiente ecuación que representa este modelo de precios:

$$
P=\left(\mathrm{I}-A^{\prime}\right)^{-1} B=M^{\prime} B
$$

donde $M^{\prime}$ es la transpuesta de la matriz inversa e I es el vector de componente exógeno. Puesto que los precios se supone que son iguales a uno, los cambios en I son expresados en porcentaje. Por tanto al calcular la relación del shock con el componente exógeno se obtiene un cambio porcentual en el I-PShock- y, a continuación se estima la variación de los precios endógenos para el sector $j$ :

$$
\begin{gathered}
\Delta P_{j}=M_{j j} \text { PShock }_{j} \\
\text { PShock }_{j}=\frac{\text { Shock }}{\mathrm{I}_{j}}
\end{gathered}
$$

El cambio en el en el IPP causado por el aumento de los precios del sector j se puede calcular como:

$$
\Delta I P P_{j}=\sum_{i} \frac{P I B_{i}}{Y_{j}} M_{i j} \text { PShock }_{j}
$$

Así como la elasticidad se puede expresar como:

$$
\varepsilon_{I P P, j}=\frac{\Delta I P P_{j}}{\Delta p_{j}}
$$

A continuación se mostrará el gráfico obtenido resultado del shock realizado a la economía georgiana, concretamente se realizará un shock uniforme expresado como un porcentaje del PIB agregado. Se ha tomado un $1.75 \%$ 
Cardenete, Manuel Alejandro y Delgado, María del Carmen. Análisis de la estructura de la economía georgiana.

como porcentaje el cual se interpretará como transferencias por parte de la Agencia de Estados Unidos para el Desarrollo Internacional a este país.

FIGURA 4: IMPACTO SECTORIAL EN EL CRECIMIENTO Y LA ELASTICIDAD DEL PIB. SHOCK DE $1,75 \%$ DEL PIB AGREGADO.

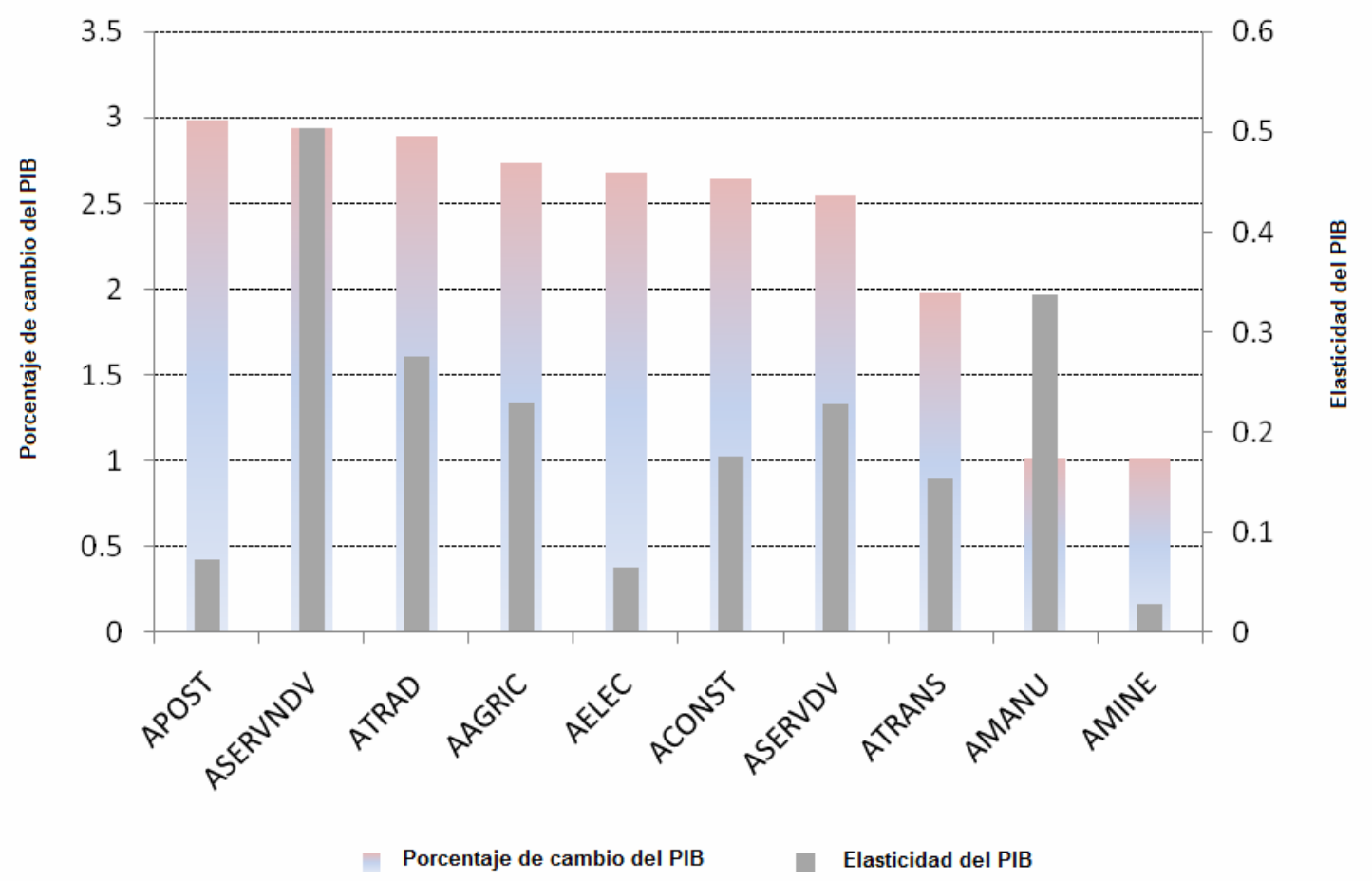

Fuente: Elaboración Propia.

Como se puede observar en la figura 4, en 2007 el sector con el mayor impacto sobre el PIB agregado fue APOST (Correos y Telecomunicaciones) con un 2.99\% de incremento en el PIB agregado, seguido de ASERVNDV (Servicios No Destinados a la Venta, incluyendo en este grupo Educación, Sanidad, Servicios sociales e Instituciones Gubernamentales) con un $2.94 \%$ de incremento y ATRAD (Comercio) con un 2.89\%. Los sectores con menor impacto son AMANU (Manufacturas) con un $1.01 \%$ y AMINE (Minería y Canteras) con un 1.01\%. En términos de elasticidad del PIB el gráfico se muestra algo diferente. El sector con mayor elasticidad es ASERVNDV (Servicios No Destinados a la Venta) con una elasticidad de $0.5 \%$, lo que significaría que un cambio de un $1 \%$ en la oferta total del sector Servicios No Destinados a la Venta provocaría un cambio en el PIB agregado de $0.5 \%$. AMANU (Manufacturas) es el sector con la segunda elasticidad más alta, un $0.34 \%$, seguido de ATRAD (Comercio) con un $0.27 \%$ y de AAGRIC con un $0.23 \%$. El sector con la menor elasticidad es AMINE (Minería y Canteras) con un $0.03 \%$, también tiene el impacto sobre el PIB más bajo.

A continuación se mostrará un gráfico que reflejará el impacto del shock de $1.75 \%$ en el precio y en la elasticidad del mismo.

En la figura 5 se puede observar como el sector APOST (Correos y Telecomunicaciones) es el que mayor impacto sobre los precios recibe con un 
Cardenete, Manuel Alejandro y Delgado, María del Carmen. Análisis de la estructura de la economía georgiana.

0.35\%, seguido por ATRAD (Comercio) con un 0.3\%, AELEC (Electricidad, Gas, Vapor y Agua Caliente) con un 0.22\%, ASERVNDV (Servicios No Destinados a la Venta) con un $0.17 \%$ y ACONST (Construcción) con un $0.15 \%$. El resto de sectores tienen un impacto sobre el precio más bajo de $0.15 \%$. En términos de elasticidades el sector con la elasticidad más alta es AMANU (Manufacturas) con un $0.55 \%$ seguido por ASERVNDV (Servicios No Destinados a la Venta) con un $0.35 \%$ y ASERVDV (Servicios Destinados a la Venta) con un $0.27 \%$. AMINE (Minería y Canteras) exhibe la más baja elasticidad de precio con un $0.03 \%$.

FIGURA 5: IMPACTO EN EL PRECIO Y LA ELASTICIDAD. SHOCK DE 1,75\% DEL PIB AGREGADO.

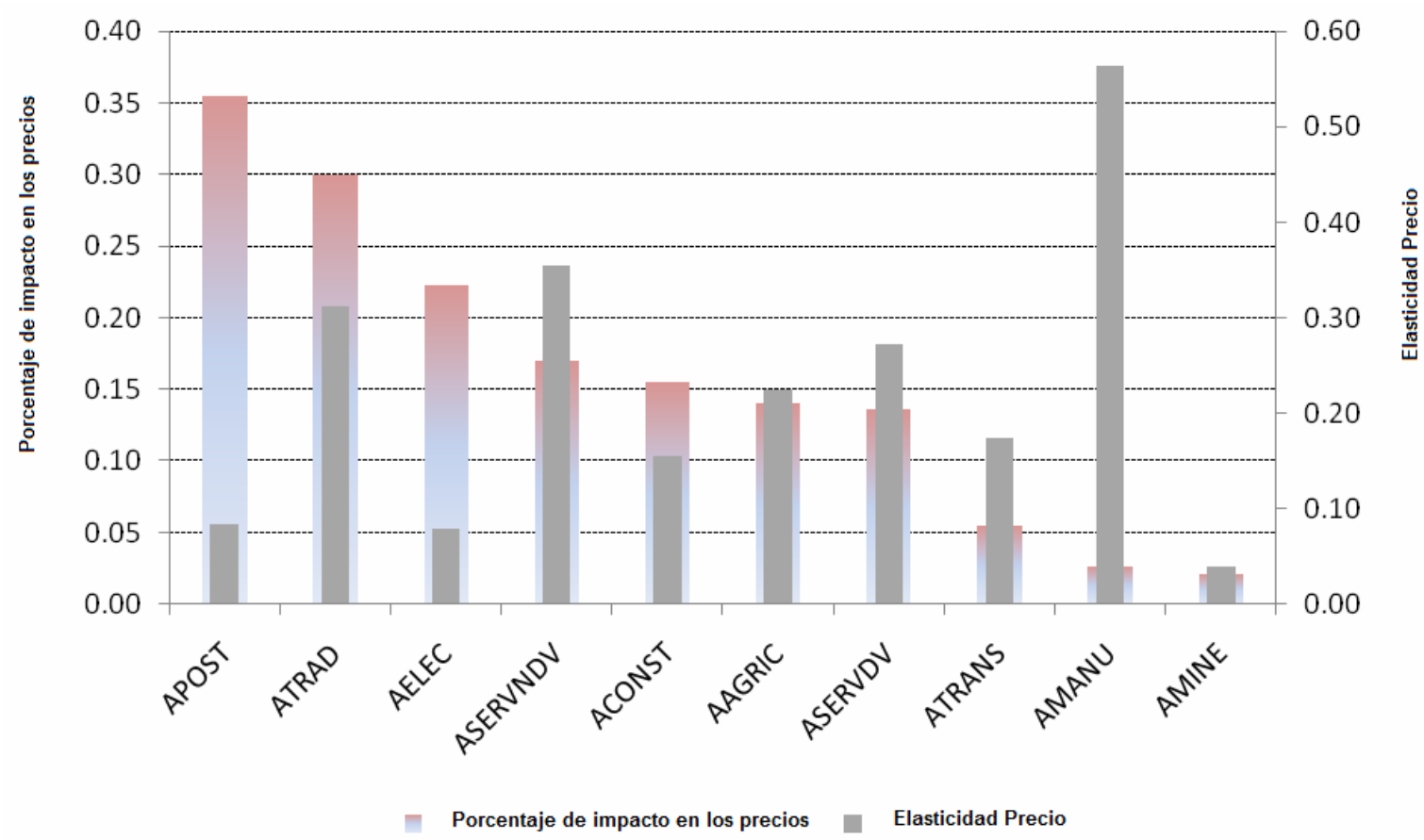

Fuente: Elaboración Propia.

\subsubsection{Simulación 2: análisis de ingresos y gastos}

Con este análisis se dará a conocer la distribución de los ingresos y gastos para los seis tipos de consumidores desagregados en la MCS. A continuación se presenta los resultados obtenidos:

TABLA 6: FUENTE DE INGRESOS COMO PORCENTAJE DEL INGRESO TOTAL.

\begin{tabular}{|l|c|c|c|c|c|}
\hline \multicolumn{1}{|c|}{ Consumidores } & Trabajo & Capital & Hogares & $\begin{array}{c}\text { Resto del } \\
\text { Mundo }\end{array}$ & Total \\
\hline URBLOWEXP & 30.14 & 51.93 & 1.80 & 16.12 & $2,309.2$ \\
\hline RURALOWEXP & 6.68 & 75.55 & 0.99 & 16.76 & $1,720.5$ \\
\hline URBMEDEXP & 28.42 & 55.75 & 0.77 & 15.05 & $3,795.5$ \\
\hline RURALMEDEXP & 11.33 & 69.03 & 1.82 & 17.80 & $2,697.6$ \\
\hline URBHIGHEXP & 15.98 & 68.93 & 0.85 & 14.22 & $3,439.4$ \\
\hline RURAHIGHEXP & 9.44 & 77.88 & 2.29 & 10.36 & $1,883.1$ \\
\hline
\end{tabular}


Cardenete, Manuel Alejandro y Delgado, María del Carmen. Análisis de la estructura de la economía georgiana.

En la tabla 6 se puede observar por filas, como el ingreso del tipo de consumidores Urbanos con nivel de gasto alto (URBHIGHEXP) está formado en un $15.98 \%$ por los salarios, un $68.93 \%$ por los rendimientos del capital, por los hogares un $0.85 \%$ y un $14.22 \%$ por transferencias del resto del mundo; el total de ingresos para este grupo de consumidores asciende a 3,439.4 millones de dólares.

TABLA 7: FUENTE DE GASTO COMO PORCENTAJE DEL GASTO TOTAL.

\begin{tabular}{|c|c|c|c|c|c|}
\hline Consumidores & $\begin{array}{c}\text { Sectores } \\
\text { Actividad }\end{array}$ & Empresa & Consumo & $\begin{array}{c}\text { Sector } \\
\text { Público }\end{array}$ & Total \\
\hline URBLOWEXP & 76.48 & 18.66 & 1.01 & 3.84 & $2,309.2$ \\
\hline RURALOWEXP & 77.77 & 19.10 & 1.54 & 1.58 & $1,720.5$ \\
\hline URBMEDEXP & 84.77 & 8.60 & 1.19 & 5.43 & $3,795.5$ \\
\hline RURALMEDEXP & 84.96 & 6.35 & 2.05 & 6.62 & $2,697.6$ \\
\hline URBHIGHEXP & 70.51 & 16.86 & 1.04 & 11.57 & $3,439.4$ \\
\hline RURAHIGHEXP & 70.85 & 12.45 & 1.24 & 15.44 & $1,883.1$ \\
\hline
\end{tabular}

En la tabla 7 por filas se puede ver como el gasto del grupo de consumidores rurales con nivel de gasto medio (RURALMEDEXP) está formado en un $84.96 \%$ por gastos destinados a las distintos sectores (agricultura, servicios, etc.), en un $6.35 \%$ por la empresa, en un $2.05 \%$ por consumo y en un $6.62 \%$ por transferencias al sector público. El total de gastos para este grupo de consumidores asciende a 2,897.6 millones de dólares.

\section{CONCLUSIONES}

Este trabajo presenta un análisis estructural de la economía georgiana. Se han analizado los sectores clave de la economía y para ello se ha utilizado la Matriz de Contabilidad Social de Georgia para el año 2007. En la aplicación de un Modelo MCS Lineal a través del análisis de multiplicadores, los resultados obtenidos clasifican los sectores de la economía georgiana según su capacidad para influir y ser influidos por cambios en ellos mismos y en el resto del sistema, en relación a unos valores medios de referencia. También se han analizado los multiplicadores de empleo de la economía.

Los resultados muestran una gran similitud con la realidad económica de Georgia, así los sectores identificados como clave para el año 2007 muestran una fiel imagen de la economía del país. Se puede resaltar como ejemplo el sector Electricidad, gas, vapor y agua caliente; este sector aparece como clave en el estudio para la economía en el año 2007. Según la literatura económica del país este sector entorpeció la economía alrededor del año 1993, debido a la falta energética del país y a la necesidad de importación de combustible y otros suministros a Rusia. Estas carencias fueron subsanadas en 2003, debido al interés del gobierno georgiano en desarrollar este sector, así resaltar, que en la actualidad, la energía hidroeléctrica llega a representar el $83 \%$ del total de la producción energética del país. 
Cardenete, Manuel Alejandro y Delgado, María del Carmen. Análisis de la estructura de la economía georgiana.

Se puede destacar también como el impacto en el PIB del shock inyectado a la economía georgiana, llevado a cabo en este estudio, ponen de manifiesto una importante contribución de la Agencia de Estados Unidos para el Desarrollo Internacional en el crecimiento económico de la región.

Finalmente, se puede concluir señalando que son muchos los cambios que la región de Georgia debe de afrontar y no son fáciles de digerir. Por ello, es necesario el mantenimiento de un reparto financiero, que aunque exigente en relación a resultados esperados, permita consolidar la recuperación de la economía, sin que turbulencias económicas puedan poner en peligro los importantes avances realizados en el largo proceso de convergencia. 
Cardenete, Manuel Alejandro y Delgado, María del Carmen. Análisis de la estructura de la economía georgiana.

\section{REFERENCIAS BIBLIOGRÁFICAS}

Cámara, A. (2008): "Estimación de la Matriz de Contabilidad Social de la Comunidad de Madrid para el año 2000", Consejería de Economía y Hacienda. Comunidad de Madrid.

Cardenete, M.A. (2009): "La Matriz de Contabilidad Social de Georgia para el año 2007", mineo.

Cardenete, M.A. y Moniche, L. (2001): "El nuevo marco Input-Output y la SAM de Andalucía para 1995", Cuadernos de Ciencias Económicas y Empresariales. Vol. 41, 13-31.

Cardenete, M.A. y Sancho, F. (2006): "Elaboración de una matriz de contabilidad social a través del método de entropía cruzada: España 1995", Estadística Española. Vol. 48, №161, 67-100.

Curbelo, J. L. (1986): "Una Introducción a las Matrices de Contabilidad Social y a su Uso en la Planificación del Desarrollo Regional", Estudios Territoriales. Vol. 7, 147-155.

Defourney, J. y Thorbeke, E. (1984): "Structural Path Analysis and Multiplier Decomposition within a Social Accounting Matrix framework", The Economic Journal. Vol. 94.

De Miguel, F.J. y Manresa, A. (2004):"Modelos SAM lineales y distribución de la renta: una aplicación para la economía extremeña", Estudios de Economía Aplicada. Vol. 22-3, 577-603.

Dietzenbacher, E., Van der Linden, J.A. y Steenge, A. (1993): "The Regional Extraction Method: EC Input-Output Comparisons", Economic Systems Research, vol. 5, 185-206.

Fernández, J. y González, P. (2004): "Matrices de Contabilidad Social: Una Panorámica", Ekonomiaz. Vol. 57, $3^{\text {er }}$ Cuatrimestre, 133-158.

Fernández, M. y Polo, C. (2001): "Una Nueva Matriz de Contabilidad Social para España: la SAM-90", Revista Estadística Española. Vol. 43, №148, 281-311.

Ferri, J. y Uriel, E. (2000): "Multiplicadores contables y análisis estructural en la Matriz de Contabilidad Social. Una aplicación al caso español", Investigaciones económicas. Vol. 24 №2, 419-453.

Ghosh, A. (1958): "Input-Output Approach in Allocation System" , Económica, vol. 25, 58-64.

Gómez Gómez-Plana, A. (2001): "Extensiones de la matriz de contabilidad social de España", Estadística Española. Vol. 43, №147, 125-163.

Kehoe, T. J.; Manresa, A.; Polo, C. y Sancho, F. (1988): "Una Matriz de Contabilidad Social de la Economía Española", Estadística Española. Vol. 30, 5-33.

Leontief, W. (1941): The Structure of American Economy, 1919-1924: an Empirical Application of Equilibrium Analysis, Harvard Univ. Press, Cambridge, Mass.

Lima, C., Cardenete, M. A. y Vallés , J. (2005): "A Structural Analysis of a Regional Economy using a Social Accounting Matrix: 1990-1999", Investigaciones Regionales. Vol. 5, 113-138.

Polo, C., Roland-Holst, D. y Sancho, F. (1991): "Descomposición de Multiplicadores en un Modelo Multisectorial: una Aplicación al Caso Español", Investigaciones Económicas. Vol. XV, №1, 53-69. 
Cardenete, Manuel Alejandro y Delgado, María del Carmen. Análisis de la estructura de la economía georgiana.

Pulido, A. y Fontela, E. (1993): Análisis input-output. Modelos, datos y aplicaciones, Editorial Pirámide, Madrid.

Pyatt, G. (1977): Social Accounting for Development Planning with Special Reference to Sri Lanka, Cambridge Univ. Press.

Pyatt, G. y Round, J.I. (1979): "Accounting and fixed price multipliers in a Social Accounting Matrix framework", The Economic Journal. Vol.89.

Robinson, S., Cattaneo, A. y El-Said, M. (2001): "Updating and Estimating a Social Accounting Matrix Using Cross Entropy Methods", Economic Systems Research. Vol. 13, nำ.

Sonis, M., Hewings, G.J.D. y Guo, D. (2000): “A new image of Classical Key Sector Analysis: Minimum information decomposition of the Leontief inverse", Economic Systems Research. Vol.12, no 3.

Stone, R. (1962): A Social Accounting Matrix for 1960 en A Programme for Growth, Chapman and Hall Ltd. (Eds.), London.

Stone, R. (1978): The Disaggregation of the Household Sector in the National Accounts, World Bank Conference on Social Accounting Methods in Development Planning, Cambridge. 